main age group was 20-24 with speed=106 cases/year, $(\mathrm{p}=0.045)$.

Conclusion There was a decreasing trend of AIDS cases and growth in HIV+, with expressive speed among young MSM. This analysis points to the need for discussions of unprotected sexual practices, combined prevention and other actions aimed to controlling the epidemic in greater vulnerability groups.

\section{P3.152 PREVALENCE OF BACTERIAL VAGINOSIS INFECTION AND ASSOCIATED FACTORS IN WOMEN WHO HAVE SEX WITH WOMEN}

MTC Duarte, MAO Ignacio, J Andrade, APF Freitas, MG Silva. São Paulo State University, Brazil

\subsection{6/sextrans-2017-053264.387}

Introduction The present research aims to fill a gap in the national and international literature concerning prevalences and factors related to Bacterial vaginosis (VB) in Women who Have Sex with Women (WSW).

Methods It is a cross sectional, analytical and non-radomized study with 128 WSW from Botucatu - SP and surrounding regions who answered the call from social media, mass communication means, health services and friends or acquaintances from January to November,2015. Data was obtained by the researchers involved in the main study, through interviews ang gynaecological exam. The diagnostic of VB was abteined through gram staining. Associations were estimated by multiple regression.

Results The prevalence of BV was $41,1 \% \%$ and factors associated were vaginal douching $[\mathrm{OR}=3,29$ (IC:95\%: 1,26-8,59) $\mathrm{p}=0014]$ and sex toys use[OR=2,34 (IC:95\%: 1,00-5,50); $\mathrm{p}=0049$ ].

Conclusion Considered as whole, these data lead to conclusion that the individuals of this study presented high vulnerability to STI/AIDS, as shown by the high prevalence of VB. This study clearly shows the need for a specific health assistance to these women, promoting prevention and education in a holistic approach.

\section{P3.153 SPATIAL AND TEMPORAL ASSOCIATIONS BETWEEN CONGENITAL SYPHILIS CASES AND EPIDEMIOLOGICAL CHARACTERISTICS OF INFECTIOUS SYPHILIS IN ENGLAND}

Martina Furegato, Helen Fifer, Hamish Mohammed, lan Simms, Louise Logan, Noel Gill, Andre Charlett, Gwenda Hughes. Public Health England, London, UK

\subsection{6/sextrans-2017-053264.388}

Introduction There has been a rapid rise in infectious syphilis (IS) diagnoses in England since 2011 but congenital syphilis (CS) is rare. In 2016, 3 CS cases were diagnosed in geographically dispersed areas of England. Unusually, their mothers had tested negative at first trimester antenatal screen (screen-negative), indicating syphilis acquisition during pregnancy. Simulation modelling using historical CS cases indicated an event probability of $3 \%$. We investigated the spatial and temporal relationship between screen-negative CS cases and IS epidemiology in the affected areas.

Methods Data from 01/2011-06/2016 were obtained from GUMCADv2, the national electronic surveillance system.
England was divided into 3 syphilis epidemiological areas (SEAs): wider incident areas (WIAs; the 3 affected and immediate surrounding counties); endemic areas (with established epidemics in men who have sex with men-MSM) and nonincident non-endemic areas (NINEAs). Time-series analysis (TSA) was used to estimate IS outliers by gender, sexual orientation and SEA. Associations between IS characteristics and SEA (WIAs vs. NINEAs) were assessed using Pearson's chisquare and Kruskal-Wallis tests. Mothers of CS cases were excluded from analyses.

Results In 2011-2016, IS rates/100,000 in WIAs rose in heterosexual women (1.3-3.0) and MSM (8.9-13.9) but fell in heterosexual men (3.7-3.0). In NINEAs, rates rose in heterosexual women (1.6-1.9), MSM (5.0-10.8) and heterosexual men (2.7-3.2). On TSA, IS cases significantly exceeded expected bounds in 2016 in heterosexual women in WIAs; no exceedance was seen in NINEAs. In 2016, heterosexual women with IS were more likely to be UK-born in WIAs than in NINEAs $(78 \%$ vs. $39 \%$; $<0.001)$. A greater proportion of MSM were bisexual in WIAs than in NINEAs $(11 \%$ vs. $8 \%$; $\mathrm{p}<0.001)$.

Conclusion Increased syphilis transmission in some sexual networks of MSM and a higher proportion of bisexual men in WIAs may have created more opportunities for IS acquisition in women. Efforts to raise awareness of the potential risk of acquiring syphilis during pregnancy are needed.

\section{P3.154 CLONAL SPREAD OF AZITHROMYCIN RESISTANT NEISSERIA GONORRHOEAE IN CANADA (2014-2015)}

${ }^{1}$ I Artin, ${ }^{1} \mathrm{P}$ Sawatzky, ${ }^{2} \mathrm{~B}$ Lefebvre, ${ }^{3} \mathrm{~V}$ Allen, ${ }^{4} \mathrm{P}$ Naidu, ${ }^{5} \mathrm{~L}$ Hoang, ${ }^{6} \mathrm{G}$ Horsman, ${ }^{1}$ MR Mulvey. ${ }^{1}$ Public Health Agency of Canada, Winnipeg, Canada; 'Laboratoire De Santé Publique Du Québec, Ste-Anne-De-Bellevue, Canada; ${ }^{3}$ Public Health Ontario, Toronto, Canada; ${ }^{4}$ Alberta Health Services, Edmonton, Canada; ${ }^{5}$ British Columbia Centres For Disease Control, Vancouver, Canada; ${ }^{6}$ Saskatchewan Disease Control Laboratory, Regina, Canada

\subsection{6/sextrans-2017-053264.389}

Introduction Neisseria gonorrhoeae have acquired resistance to many antimicrobials including third generation cephalosporins and azithromycin, which are the current co-therapy recommended by the Canadian STI guidelines for gonorrhoea treatment. Minimum inhibitory concentrations (MIC) to azithromycin and molecular sequence types were determined for N. gonorrhoeae circulating in Canada.

Methods Between 2014 and 2015, N. gonorrhoeae strains isolated by Canadian provincial public health laboratories were submitted to the National Microbiology Laboratory (NML) $(n=4,720)$ for azithromycin MIC determination (resistance MIC $\geq 2.0 \mathrm{mg} / \mathrm{L}$ ) by agar dilution. N. gonorrhoeae multi-antigen sequence typing (NG-MAST) was used for molecular typing.

Results Azithromycin resistance was identified in 3.3\% (127/ $3,809)$ and 4.7\% (198/4,190) of N. gonorrhoeae in 2014 and 2015, respectively, a significant increase since $2013(\mathrm{p}<0.001)$. MICs ranged from 2 to $16 \mathrm{mg} / \mathrm{L}$. The most common sequence types identified in 2014 were ST10451 $(n=40)$, ST10567 $(\mathrm{n}=38)$ and ST11765 $(\mathrm{n}=10)$. ST11765 is closely related to ST10451, differing by $1 \mathrm{bp}$ in the por allele. In 2015, the prevalent sequence types were ST12302 $(n=110)$, ST10451 $(n=34)$ and ST9047 $(n=23)$. ST10451, identified in Quebec, Ontario and Alberta, was newly identified in 2014 and also identified in 2015. ST10451 is related to ST1407 (differing by $1 \mathrm{bp}$ in the por allele) which is an internationally- 